Editor's Note: These short, critical reviews of recent papers in the Journal, written exclusively by graduate students or postdoctoral fellows, are intended to summarize the important findings of the paper and provide additional insight and commentary. For more information on the format and purpose of the Journal Club, please see http://www.jneurosci.org/misc/ifa_features.shtml.

\title{
Nrg1 Reverse Signaling in Cortical Pyramidal Neurons
}

\author{
Sandra Peregrin Pedrique and Pietro Fazzari \\ Instituto de Neurociencias, Consejo Superior de Investigaciones Cientıficas and Universidad Miguel Hernandez, 03550 Sant Joan d'Alacant, Spain \\ Review of Chen et al.
}

Schizophrenia is a severe neurological disorder characterized by psychotic symptoms, emotional distress, and cognitive deficits. It results from an amalgam of genetic, developmental, and environmental factors. In the last decade, several studies identified Nrg1 and its receptor ErbB4 as major schizophrenia-risk genes (Stefansson et al., 2002; Norton et al., 2006). Moreover, behavioral studies on different Nrg1 and ErbB4 mutant mice revealed various endophenotypes that are believed to be useful for modeling schizophrenia in mice (e.g., hyperactivity in response to novelty, hypersensitivity to psychostimulants, and deficits in working memory tasks). Similar phenotypes were shown in mice expressing mutated BACE-1 (the extracellular Nrg1cleavage enzyme) (Savonenko et al., 2008) or Aph1B/C- $\gamma$-secretase (the Nrg1 intracellular proteolytic enzyme) (Dejaegere et al., 2008). Together, these findings suggest that perturbed Nrg1 signaling leads to functional deficits that correlate with schizophrenia. Nonetheless, Nrg1 and ErbB4 mutants do not display overt morphological defects in the brain and the precise role of $\mathrm{Nrg} 1 /$ ErbB4 signaling in the nervous system remains elusive.

In addition to canonical forward signaling via ErbB family receptors, the membrane-bound form of Nrg1 can also activate reverse signaling. Upon synaptic activation or receptor binding, membrane-

Received Sept. 6, 2010; revised Sept. 30, 2010; accepted Oct. 5, 2010. P.F. is recipient of a Marie Curie Intra European Fellowship.

Correpondence should be addressed to Pietro Fazzari, Via Graglia 57, 13898 0cchieppo Superiore (BI), Italy. E-mail: p.fazzari@yahoo.it. DOI:10.1523/JNEUROSCI.4669-10.2010

Copyright $\odot 2010$ the authors $\quad 0270-6474 / 10 / 3015005-02 \$ 15.00 / 0$ associated type III Nrg1 is cleaved by $\gamma$-secretase to release the intracellular domain (NRG1-ICD), which translocates to the nucleus where it regulates gene expression (Bao et al., 2003, 2004). Recently, it was shown that Nrg1/ErbB4 forward signaling promotes the formation of inhibitory circuits in the cortex (Fazzari et al., 2010). Chen et al. (2010) show that Nrg1 reverse signaling is important for the development of the dendritic arbor of pyramidal neurons (PNs).

To address the function of type III Nrg1 in PNs in vivo, the authors compared the morphology of dendritic arbor of neurons in type III Nrg1-deficient and wild-type (WT) mice. The histological analysis of mutant and control brains revealed that the total length and number of branches of basal dendrites were greatly reduced in type III Nrg1-deficient versus WT neurons. Because axon length could not be measured efficiently in vivo, the authors used primary cortical cultures. Mimicking the in vivo data, the type III Nrg1 mutant neurons showed a clear reduction in dendritic development in vitro. In addition, the axonal length, but not the axonal branching, was reduced in type III Nrg1-deficient neurons.

To elucidate the molecular mechanisms involved in type III NRG1 function in the development of dendrites and axons, Chen et al. (2010) verified that Nrg1 reverse signaling occurs in cortical cultures by showing that ErbB4 binding stimulated translocation of NRG1-ICD to the nucleus and activated transcription of a luciferase reporter gene. Furthermore, they found mutations in the intracellular portion of Nrg1 (V321A/V322A and K329A/Q330A) that abolished the transcriptional activation. The actual translocation of control and mutant NRG1-ICD to the nucleus was also measured by immunofluorescence, confirming the results of the luciferase assay. Consistent with this finding, a similar mutation (V321L) that is associated with schizophrenia (Walss-Bass et al., 2006) was previously shown to impair $\gamma$-secretase cleavage of Nrg1 (Dejaegere et al., 2008).

Interestingly, the immunofluorescence shows the presence of NRG1-ICD in the nucleus even without ErbB4 stimulation. Consistently, in the luciferase assay, WT Nrg1 displays a basal level of transcriptional activation in absence of exogenous ErbB4 that is higher than the uncleavable forms. Although Chen et al. (2010) do not clarify whether this difference is statistically significant, these experiments suggest that a small but considerable amount of WT Nrg1 was processed in absence of exogenous ErbB4 stimulation. As the authors subsequently claim that type III Nrg1 function is ErbB4 independent, it would be important to clarify this issue.

Next, Chen et al. (2010) tried to rescue the dendritic and axonal phenotype in type III Nrg1-null neurons by expressing WT or mutant forms of type III Nrg1 in vitro. They found that both residues V321/V322 and K329/Q330, which are required for type III Nrg1 cleavage and nuclear translocation, were necessary to rescue the dendritic phenotype. In contrast, even the uncleavable Nrg1 mutants could rescue the axonal phenotype, show- 
ing that Nrg1-ICD processing is not essential for axonal maturation.

Because Nrg1-ICD transcriptional activation is triggered by receptor binding, the authors asked whether type III Nrg1 actions were dependent on endogenous ErbB4. Intriguingly, the authors showed that pyramidal neurons from ErbB4deficient mice displayed normal morphology. This result suggests that ErbB4 plays a redundant role in Nrg1 backward signaling, but it still leaves open the possibility that another receptor of the ERBB family might activate Nrg1-ICD in cortical neurons (Fox and Kornblum, 2005). To study this issue, we believe that it would be valuable to quantify the amount of Nrg1 that is cleaved to release Nrg1ICD in ErbB4 mutant cultures. In addition, it would be informative to test the effect of exogenous ErbB4 stimulation on dendritic arborization; if the amount of Nrg1-ICD transcriptional activation proportionally increases dendritic arborization, exogenous ErbB4 stimulation is expected to enhance dendritic development in a dose-dependent manner. Alternatively, Nrg1-ICD activity could be just a permissive factor. In this case, a low level of Nrg1-ICD transcriptional activation might be necessary and sufficient to allow proper dendritic development, even in absence of ErbB4.

In summary, the work by Chen et al. (2010) provides important evidence that the Nrg1 is differentially required in PNs for axonal and dendritic development. In particular, Nrg1 reverse signaling, initiated by $\gamma$-secretase-mediated intracellular cleavage, is necessary for dendritic development of cortical neurons both in vitro and in vivo. This suggests a dual function for Nrg1 in sculpting the cortical wiring. On one hand, Nrg1 forward signaling via ErbB4, which is mainly expressed in parvalbumin-positive interneurons that target the soma and the axonal initial segment of PNs, promotes the establishment of negative feedback inhibitory circuits on PNs (Fazzari et al., 2010). In particular, ErbB4 is required both for the formation of excitatory synapses onto parvalbuminpositive interneurons and for the formation of inhibitory synapses over cortical pyramidal cells. On the other hand, Chen and colleagues (2010) suggest that reverse signaling via Nrg1 shapes cortical cir- cuitry by promoting the development of PNs dendrites.

Nonetheless, several issues remain to be elucidated about the role of Nrg1-ICD in vivo. First, the histology of the brains of type III Nrg1-deficient mice should be carefully analyzed to explain the surprising finding that, despite a dramatic decrease in PN dendritic length ( $\sim 50 \%)$, these mutant mice do not display a decrease in cortical volume. Notably, a decreased cortical volume is a typical hallmark of schizophrenia. Second, since Nrg1-ICD regulates the expression of PSD-95, a major postsynaptic scaffolding protein of excitatory synapses (Bao et al., 2004), it would be useful to determine whether type III Nrg1-deficient mice display defects in synaptogenesis and/or in excitatory transmission between PNs. Third, it was reported that Nrg1-ICD prevents neuronal apoptosis by regulating the expression of apoptotic genes (Bao et al., 2003). This introduces a caveat to the Chen et al. (2010) study since the impaired neuritic development might also be explained by distress resulting from an increased sensitivity to apoptotic factors. Finally, the molecular mechanisms of Nrg1 reverse signaling remain to be clarified. By definition, reverse signaling is elicited by the intracellular domain of a ligand upon binding to its receptor. However, the authors claim that Nrg1 backward signaling is independent of ErbB4 and of ERBB receptors kinase activity. It was previously shown that $\gamma$-secretasedependent cleavage of type III Nrg1 can also be activated by synaptic activity (Bao et al., 2004). Nevertheless, synaptic activity hardly explains the in vitro phenotype at $3 \mathrm{~d}$ in vitro reported by Chen et al. (2010) because synaptic activity is not yet present in these young cultures. In addition, the fact that uncleavable Nrg1 expression in type III Nrg1-deficient PNs rescues the axonal phenotype suggests a cell-autonomous function that is independent of Nrg1-ICD transcriptional activity.

In conclusion, Chen and colleagues (2010) provide convincing evidence that type III Nrg1 reverse signaling promotes dendritic arborization in cortical PNs. Remarkably, this Nrg1-ICD function seems independent of ErbB4, suggesting a heterodox form of backward signaling. These findings support the relevance of
Nrg1 function in cortical development; nonetheless, more work remains to be done to understand the role of Nrg1 in cortical wiring and in the etiology of schizophrenia.

\section{References}

Bao J, Wolpowitz D, Role LW, Talmage DA (2003) Back signaling by the Nrg-1 intracellular domain. J Cell Biol 161:1133-1141.

Bao J, Lin H, Ouyang Y, Lei D, Osman A, Kim TW, Mei L, Dai P, Ohlemiller KK, Ambron RT (2004) Activity-dependent transcription regulation of PSD-95 by neuregulin-1 and Eos. Nat Neurosci 7:1250-1258.

Chen Y, Hancock ML, Role LW, Talmage DA (2010) Intramembranous valine linked to schizophrenia is required for neuregulin 1 regulation of the morphological development of cortical neurons. J Neurosci 30:9199-9208.

Dejaegere T, Serneels L, Schäfer MK, Van Biervliet J, Horré K, Depboylu C, Alvarez-Fischer D, Herreman A, Willem M, Haass C, Höglinger GU, D’Hooge R, De Strooper B (2008) Deficiency of Aph1B/C-gamma-secretase disturbs Nrg1 cleavage and sensorimotor gating that can be reversed with antipsychotic treatment. Proc Natl Acad Sci U S A 105:9775-9780.

Fazzari P, Paternain AV, Valiente M, Pla R, Luján R, Lloyd K, Lerma J, Marín O, Rico B (2010) Control of cortical GABA circuitry development by Nrg1 and ErbB4 signalling. Nature 464:1376-1380.

Fox IJ, Kornblum HI (2005) Developmental profile of ErbB receptors in murine central nervous system: implications for functional interactions. J Neurosci Res 79:584-597.

Norton N, Moskvina V, Morris DW, Bray NJ, Zammit S, Williams NM, Williams HJ, Preece AC, Dwyer S, Wilkinson JC, Spurlock G, Kirov G, Buckland P, Waddington JL, Gill M, Corvin AP, Owen MJ, O'Donovan MC (2006) Evidence that interaction between neuregulin 1 and its receptor erbB4 increases susceptibility to schizophrenia. Am J Med Genet B Neuropsychiatr Genet 141:96-101.

Savonenko AV, Melnikova T, Laird FM, Stewart KA, Price DL, Wong PC (2008) Alteration of BACE1-dependent NRG1/ErbB4 signaling and schizophrenia-like phenotypes in BACE1-null mice. Proc Natl Acad Sci U S A 105:5585-5590.

Stefansson H, Sigurdsson E, Steinthorsdottir V, Bjornsdottir S, Sigmundsson T, Ghosh S, Brynjolfsson J, Gunnarsdottir S, Ivarsson O, Chou TT, Hjaltason O, Birgisdottir B, Jonsson H, Gudnadottir VG, Gudmundsdottir E, Bjornsson A, Ingvarsson B, Ingason A, Sigfusson S, Hardardottir H, et al. (2002) Neuregulin 1 and susceptibility to schizophrenia. Am J Hum Genet 71:877-892.

Walss-Bass C, Liu W, Lew DF, Villegas R, Montero P, Dassori A, Leach RJ, Almasy L, Escamilla M, Raventos H (2006) A novel missense mutation in the transmembrane domain of neuregulin 1 is associated with schizophrenia. Biol Psychiatry 60:548-553. 